\title{
LOS ÁFIDOS (Hemiptera: Aphididae) EN EL CALLEJÓN DE HUAYLAS - ANCASH, PERÚ
}

\section{APHIDS (Hemiptera: Aphididae) AT THE CALLEJÓN DE HUAYLAS - ANCASH, PERÚ}

\author{
Karina Vilca Mallqui ${ }^{1}$ y Clorinda Vergara Cobián ${ }^{2}$
}

\begin{abstract}
Resumen
Se colectaron áfidos en plantas cultivadas y malezas en el Callejón de Huaylas, Ancash. Se realizaron micropreparados y se determinaron 31 especies de áfidos ubicadas en 4 subfamilias y 5 tribus. Se estableció 123 asociaciones áfidos-planta, en 52 especies de plantas en 45 géneros de 19 familias. Se registran por primera vez en la localidad las siguientes 18 especies de áfidos: Acyrtosiphon kondoi, Aphis cytisorum, A. fabae, A. helianthi, A. nerii, A. spiraecola, Brachycaudus schwartzi, Capitophorus elaeagni, Hyalopterus pruni, Metopolophium dirhodum, Myzus ornatus, Nasonovia ribisnigri, Rhopalosiphoninus latysiphon, Rhopalosiphum padi, Tuberolachnus salignus, Uroleucon ambriosae, U. sonchi y Wahlgreniella nervata y se cita por primera vez para el Perú a Aphis helianthi y Aphis cytisorum. Se confirmó el carácter polífago de Aphis craccivora, A. gossypii, A. fabae, A. spiraecola, Brachycaudus helichrysi, Macrosiphum euphorbiae, Myzus ornatus, M. persicae, Uroleucon ambrosiae. Brevicoryne brassicae mostró un amplio rango de hospederos. Se confirmó a Aphis cytisorum como el áfido que afecta a la retama en el Callejón de Huaylas, conjuntamente con Aphis craccivora y A. fabae, y la especificidad de $A$. helianthi para especies de la familia Asteraceae.
\end{abstract}

Palabras clave: áfidos, hospedantes, relación áfido - planta.

\begin{abstract}
Plant aphids were collected in different crop plants and weeds at the Callejón de Huaylas zone, Ancash, Perú. Micro slides were prepared for species determination, and 31 aphid species corresponding to 4 subfamilies and 5 tribes were identified. 123 aphid-plant relationships were established in 52 plant species from 45 genera and 19 families The following 18 aphid species are registered for the first time in the Callejón de Huaylas: Acyrtosiphon kondoi, Aphis cytisorum, A. fabae, A. helianthi, A. nerii, A. spiraecola, Brachycaudus schwartzi, Capitophorus elaeagni, Hyalopterus pruni, Metopolophium dirhodum, Myzus ornatus, Nasonovia ribisnigri, Rhopalosiphoninus latysiphon, Rhopalosiphum padi, Tuberolachnus salignus, Uroleucon ambriosae, U. sonchi y Wahlgreniella nervata and Aphis helianthi as well as Aphis cytisorum are registered for the first time in Peru. The oligophagous character of Aphis craccivora, A. gossypii, A. fabae, A. spiraecola, Brachycaudus helichrysi, Macrosiphum euphorbiae, Myzus ornatus, M. persicae, Uroleucon ambrosiae was confirmed. Brevicoryne brassicae showed and a wide host range. Aphis cytisorum was confirmed as the aphid affecting the retama plant at the Callejón de Huaylas, together with Aphis craccivora y A. fabae as well as the specificity of A. helianthi for plant species of the Asteraceae family.
\end{abstract}

Key words: aphid, hosts, aphid - plant relationships.

\section{Introducción.}

El Callejón de Huaylas, que comprende territorios de las provincias de Huaraz, Yungay, Carhuaz y Huaylas, es una de las zonas agrícolas más importantes de la región Ancash y de la sierra peruana, donde se producen diferentes cultivos como maíz, papa, cebada, trigo; frutales como manzano, durazno y palto, cuyos productos son destinados al autoconsumo, los mercados locales y a las grandes ciudades como Huaraz y Lima, y flores que son exportadas a diferentes países (Ministerio de Agricultura, 2007).

Los miembros de la familia Aphididae (Insecta: Hemíptera), conocidos como pulgones o áfidos, son insectos de amplia distribución geográfica, principalmente en las regiones templadas; infestan toda clase de vegetales y muchas especies son de gran importancia agrícola, por los daños directos que causan durante su alimentación (Klingauf, 1987; Quisenbery \& Xinzhi, 2007) y especialmente por constituir el grupo de insectos más importante en la 
transmisión de virus fitopatógenos (Katis et al., 2007), varios de los cuales son importantes en el Perú y específicamente en la región Ancash (Fribourg, 2007).

En el Callejón de Huaylas se han realizado muy pocos estudios sobre la ocurrencia de especies de áfidos que se encuentran afectando la vegetación de esta zona (Ortiz et al., 1981; Vilca \& Reyes, 1999), por lo tanto, no se cuenta con un registro de las especies de áfidos en los cultivos de esta zona; conocimiento que serviría de base para desarrollar estrategias de manejo integrado de estas plagas y, por consiguiente, de las enfermedades virósicas transmitidas por estas especies.

El presente estudio se realizó con el objetivo de contribuir en la determinación de especies de la familia Aphididae del Callejón de Huaylas, elaborando una clave dicotómica para la identificación de las especies encontradas.

\section{Materiales y métodos.}

El área de trabajo de la presente investigación abarcó la zona del Callejón de Huaylas, comprendida entre las localidades de Mato, en la provincia de Huaylas, provincias de Carhuaz y Yungay, hasta Olleros en la provincia de Huaraz; ubicada entre los paralelos $9^{\circ}$ $00^{\prime}$ y $10^{\circ} 00^{\prime}$ de latitud sur y entre los meridianos $77^{\circ}$ $30^{\prime}$ y $78^{\circ} 00^{\prime}$ de longitud oeste.

Para la colecta se procedió de acuerdo a Eastop \& Van Emden (1972) y Sánchez \& Vergara (2002). Se visitaron campos en toda la zona y se evaluó la presencia de áfidos en plantas en diferentes estados de desarrollo. Se tomaron muestras constituidas por los órganos afectados por colonias de áfidos, colocándolas en bolsas de papel kraft y luego se identificaron con los siguientes datos: lugar, fecha, altitud, hospedero, entre otros, y se colocaron en frascos de vidrio con alcohol al $70 \%$. Otra parte de las muestras se colectaron directamente en alcohol al $70 \%$.

Para el montaje de los áfidos se ajustaron las metodologías descritas por Eastop y Van Emden (1972), Heikinheimo (1988), Remaudière (1992) y Voegtlin et al. (2003), al siguiente procedimiento, que mostró ser eficiente para el tratamiento de los especímenes colectados: Se seleccionaron adultos alados y ápteros, se les hizo una punción en el primer ventrito y se colocaron en un tubo de ensayo con alcohol al $70 \%$, en baño maría por 2 a 3 minutos, luego se pasaron a $\mathrm{KOH}$ al $10 \%$ en baño maría por 2 a 5 minutos. Pasado el tiempo se transfirieron a agua destilada por 2 horas, luego se cambió el agua, dejándolos en ella por 12 horas. Se transfirieron y dejaron en cloralfenol por 24 horas. Los especímenes así tratados fueron colocados en alcohol al $95 \%$ por 2 a 3 minutos. Se decantó del alcohol y pasaron a ácido acético glacial por 5 minutos, para luego colocarlos en alcohol $95 \%$ por 2 a 3 minutos, transfiriéndose a eugenol (aceite de clavo) por 10 minutos. Se procedió al montaje sobre lámina portaobjeto en bálsamo de
Canadá, cubriendo con un cubreobjeto. Cada lámina así preparada se le colocó una etiqueta con su código de identificación y se llevó a una estufa por cinco días para el secado correspondiente.

La determinación de especies se realizó en el Laboratorio de Entomología de la Unidad del Centro de Diagnóstico de Sanidad Vegetal del Servicio Nacional de Sanidad Agraria del Perú, mediante el examen de los especímenes aclarados y montados, siguiendo las claves taxonómicas y descripciones de Blackman \& Eastop (1984, 1994, 2006 y 2007), Cermeli (1984), Holman (1974), Heie (1980, 1982 y 1986), Pike et al. (2003); Voegtlin et al. (2003). Determinadas las especies, se procedió a la identificación de la lámina y se tomaron fotografías de los áfidos y sus estructuras más características. La colección de láminas micropreparados de las especies de áfidos se depositó en el Museo de Entomología "Klaus Raven Büller" de la Universidad Nacional Agraria La Molina.

\section{Resultados.}

Especies de áfidos presentes en el callejón de Huaylas.

De las 269 muestras, se determinaron 31 especies de áfidos, en 55 especies de plantas, pertenecientes a 45 géneros y 19 familias. En algunas especies de plantas se determinaron dos y hasta tres especies diferentes de áfidos.

Los áfidos determinados, pertenecen a 4 subfamilias, 5 tribus y 19 géneros, de acuerdo a la clasificación de Remaudière \& Remaudière (1997). De ellos, 16 géneros y 28 especies en la subfamilia Aphidinae, 4 géneros y 11 especies en la tribu Aphidini y, 12 géneros y 17 especies en la tribu Macrosiphini; en la subfamilia Phemphiginae, en la tribu Eriosomatini 1 género y 1 especie; en la subfamilia Myzocallidinae, en la tribu Myzocallidini 1 género y 1 especie y en la subfamilia Lachninae en la tribu Lachnini 1 género y 1 especie. Se presenta la relación de áfidos determinados, sinonimia, hospederos y lugares donde fueron encontrados, considerando la localidad y provincia.

\section{SUBFAMILIA APHIDINAE}

\section{Aphis craccivora Koch 1854}

Distribución y Hospederos: Huaraz; Carhuaz; Yungay y Huaylas. Vicia faba "haba", Medicago sativa "alfalfa", Persea americana "palto", Spartium junceum "retama", Gladiolus sp "gladiolo", Piper angustifolium "matico", Phaseolus sp.

En retama estuvo asociado a $A$. cytisorum y $A$. fabae. Citada para el Callejón de Huaylas por Vilca \& Reyes (1999), sobre palto. En el Perú ha sido registrado por Alata (1973), Valencia \& Cárdenas (1973), Valencia et al. (1975), Ortiz (1980), Da Silva et al. (1980), Nuñez (1993), Grados \& Ortiz (2004) y Delfino (2005) en diversos hospederos.

Aphis cytisorum Hartig 1841 
Distribución y Hospederos: Huaraz; Carhuaz; Yungay y Huaylas. Spartium junceum "retama"

A cytisorum fue encontrado como monófago en retama, tal como lo establecen Heie (1986), Blackman $\&$ Eastop (2006) y Holman (2009).

\section{Aphis fabae Scopoli 1763}

Distribución y Hospederos: Huaraz; Carhuaz y Huaylas. Tagetes minuta "huacatay", Solanum tuberosum "papa", Vicia faba "haba", Spartium junceum "retama", Nicotiana tabacum "tabaco silvestre".

En el Perú ha sido citado por Alata (1973), Grados \& Ortiz (2004) y Delfino (2005) en diversos hospederos.

\section{Aphis gossypii Glover 1877}

Distribución y Hospederos: Huaraz; Carhuaz y Yungay. Tagetes elíptica "chincho", Persea americana "palto", Cyphomandra betacea "sachatomate", Malus domestica "manzano", Hibiscus rosa-sinensis "cucarda", Rosa sp. "rosa".

Fue registrada para el Callejón de Huaylas por Vilca \& Reyes (1999). Citada para el Perú por Wille (1943), Alata (1973), Valencia \& Cárdenas (1973), Valencia et al. (1975), Ortiz (1980), Da Silva et al. (1980), Grados \& Ortiz (2004) y Delfino (2005) en diversos hospederos.

\section{Aphis helianthi Monell 1879}

Distribución y Hospederos: Huaraz. Tagetes elíptica "chincho", Helianthus annuus"girasol".

Fue encontrado en girasol y chincho, demostrando su especificidad para especies de la familia Asteraceae.

Aphis nerii Boyer de Fonscolombe 1841

Distribución y Hospederos: Huaraz; Carhuaz; Yungay y Huaylas. Euphorbia sp. "lechera".

En el Perú ha sido citada por Alata (1973), Valencia \& Cárdenas (1973), Ortiz (1980), Da Silva et al. (1980) y Delfino (2005), en especies de las familias Asclepiadaceae y Euphorbiaceae.

\section{Aphis spiraecola Patch 1914}

Distribución y Hospederos: Huaraz; Carhuaz; Yungay y Huaylas. Citrus limetta "lima"; Malus domestica "manzano"; Citrus jambhiri "limón rugoso"; Cydonia oblonga "membrillo"; Schifflera sp. "chiflera"; Citrus sinensis "naranjo" Ipomoea sp.; Hibiscus rosa-sinensis "cucarda"; Persea americana "palto".

En el Perú ha sido citada por Alata (1973), Valencia \& Cárdenas (1973), Valencia et al. (1975), Ortiz (1980), Da Silva et al. (1980), Grados \& Ortiz (2004) y Delfino (2005), en diversos hospederos.

\section{Hyalopterus pruni (Geoffroy 1762)}

Distribución y Hospederos: Yungay y Huaylas. Phragmites australis "carrizo".

En el Perú ha sido citado por Valencia \& Cárdenas (1973), Grados \& Ortiz (2004) y Delfino (2005), sobre carrizo y tabaco silvestre.

Rhopalosiphum maidis (Fitch 1856)
Distribución y Hospederos: Huaraz; Carhuaz; Yungay y Huaylas. Zea mays "maíz"; Hordeum vulgare "cebada".

En el caso del maíz, se encontró junto con $R$. padi. Esta especie fue registrada para el Callejón de Huaylas por Vilca \& Reyes (1999), sobre maíz. En el Perú es mencionada por Alata (1973), Ortiz (1980), Da Silva et al. (1980), Ortiz et al. (1981), Grados \& Ortiz (2004) y Delfino (2005), en diversas especies de poaceas y por Valencia \& Cárdenas (1973), en tabaco silvestre.

Rhophalosiphum padi (Linnaeus 1758)

Distribución y Hospederos: Huaraz; Carhuaz; Yungay y Huaylas. Zea mays "maíz"; Prunus persica "duraznero".

En el caso del maíz, se encontró junto con $R$. maidis. Citada para el Perú por Valencia \& Cárdenas (1973), Grados \& Ortiz (2004) y Delfino (2005) en varias especies de poaceas y Asclepias curassavica de la familia Asclepiadaceae.

Toxoptera citricidus (Kirkaldy 1907)

Distribución y Hospederos: Huaraz; Carhuaz; Yungay y Huaylas. Citrus sinensis "naranjo"; Citrus limetta "lima" y Citrus jambhiri "limón rugoso".

Fue registrada para el Callejón de Huaylas por Vilca \& Reyes (1999). Alata (1973), Valencia \& Cárdenas (1973), Ortiz (1980), Da Silva et al. (1980), Grados \& Ortiz (2004) y Delfino (2005) la citan para el Perú, en diversas especies de la familia Rutaceae, especialmente del género Citrus.

Acyrthosiphon kondoi Shinji 1938

Distribución y Hospederos: Carhuaz. Medicago sativa "alfalfa".

En el Perú fue citada por Ortiz \& Raven (1990), Grados \& Ortiz (2004) y Delfino (2005).

Acyrthosiphon pisum (Harris 1776)

Distribución y Hospederos: Huaraz; Carhuaz y Yungay. Medicago sativa "alfalfa"; Pisum sativum "arveja".

Para el Callejón de Huaylas fue citada por Vilca \& Reyes (1999). Citada para el Perú por Alata (1973), Valencia et al. (1975), Da Silva et al. (1980), Grados \& Ortiz (2004) y Delfino (2005) sobre diversas especies de Fabaceae.

Brachycaudus helichrysi (Kaltenbach 1843)

Distribución y Hospederos: Huaraz; Yungay y Huaylas. Solanum tuberosum "papa"; Zea mays "maíz"; Prunus persica "duraznero"; Rosa sp. "rosal"; Helianthus annuus "girasol".

Fue registrada para el Callejón de Huaylas por Vilca \& Reyes (1999). En el Perú fue citada por Alata (1973), Valencia et al. (1975), Grados \& Ortiz (2004) y Delfino (2005) en diversos hospederos.

Brachycaudus schwartzi (Börner 1931)

Distribución y Hospederos: Huaraz; Carhuaz; Yungay y Huaylas. Solanum tuberosum "papa"; Prunus persica "duraznero" y Medicago sativa "alfalfa". 
En el Perú ha sido registrada por Díaz \& Lozada (2007) en duraznero.

\section{Brevicoryne brassicae Linnaeus 1758}

Distribución y Hospederos: Huaraz; Carhuaz; Yungay y Huaylas. Medicago sativa "alfalfa"; Pisum sativum "arveja"; Solanum tuberosum "papa" Tagetes elíptica "chincho"; Brassica oleracea "repollo"; Brassica oleracea var. botrytis "coliflor"; Brassica rapa "nabo"; Lactuca sativa "lechuga"; Nicotiana tabacum "tabaco silvestre".

Esta especie fue registrada por Vilca \& Reyes (1999) para el Callejón de Huaylas sobre repollo. En el Perú ha sido citada por Wille (1943), Alata (1973), Valencia \& Cárdenas (1973), Valencia et al. (1975), Da Silva et al. (1980), Grados \& Ortiz (2004) y Delfino (2005) en diversos hospederos.

Capitophorus elaeagni (Del Guercio 1894)

Distribución y Hospederos: Carhuaz y Huaylas. Rosa sp. "rosa"; Cynara scolymus "alcachofa" y Bouganvillea spectabilis "buganvilia".

En el Perú ha sido mencionada por Alata (1973), Valencia et al. (1975) y Delfino (2005), en alcachofa.

Macrosiphum euphorbiae (Thomas 1878)

Distribución y Hospederos: Huaraz; Carhuaz; Yungay y Huaylas. Zea mays "maíz"; Rumex crispus "lengua de vaca"; Solanum tuberosum "papa"; Medicago sativa "alfalfa" Triticum aestivum "trigo"; Malva sylvestris "malva"; Pisum sativum "arveja"; Tagetes minuta "huacatay"; Rosa sp. "rosal"; Ruta graveolens "ruda"; Prunus persica "duraznero"; Lactuca sativa "lechuga"; Dahlia sp. "dalia"; Hibiscus rosa-sinensis "cucarda"; Cynara scolymus "alcahofa"; Phaseolus vulgaris "frijol"; Bouganvillea spectabilis "buganvilia"; Physalis sp. e Ipomoea sp.

Esta especie fue citada por Vilca \& Reyes (1999) para el Callejón de Huaylas en repollo. Alata (1973), Valencia \& Cárdenas (1973), Valencia et al. (1975), Grados \& Ortiz (2004) y Delfino (2005), lo mencionan para el Perú en diversos hospederos.

Macrosiphum rosae (Linnaeus 1758)

Distribución y Hospederos: Huaraz; Carhuaz y Huaylas. Rosa sp. "rosal".

Esta especie fue mencionada por Vilca \& Reyes (1999) para el Callejón de Huaylas en rosal. Citada para el Perú por Alata (1973), Valencia et al. (1975), Grados \& Ortiz (2004) y Delfino (2005) en Rosa spp.

Metopolophium dirhodum (Walker 1849)

Distribución y Hospederos: Huaraz. Hordeum vulgare "cebada".

Fue citada para el Perú por Alata (1973), Valencia et al. (1975), Ortiz et al. (1981), Da silva et al. (1980), Grados \& Ortiz (2004) y Delfino (2005) en diversas especies de gramíneas.

Myzus ornatus Laing 1932

Distribución y Hospederos: Huaraz; Carhuaz y Yungay. Solanum tuberosum "papa"; Viola tricolor "pensamiento"; Capsicum pubescens "rocoto";
Bouganvillea spectabilis "buganvilia"; Piper angustifolium "matico"; Plantago major "llantén".

Para el Perú fue mencionada por Alata (1973), Valencia et al. (1975), Grados \& Ortiz (2004) y Delfino (2005), sobre diversos hospederos.

Myzus persicae (Sulzer 1776)

Distribución y Hospederos: Huaraz; Carhuaz; Yungay y Huaylas. Solanum tuberosum "papa"; Bidens pilosa "amor seco"; Schifflera sp. "chiflera"; Cyphomandra betacea "sachatomate"; Cynara scolymus "alcachofa"; Medicago sativa "alfalfa"; Lactuca sativa "lechuga"; Viola tricolor "pensamiento"; Persea americana "palto"; Prunus persica "duraznero"; Dahlia sp. "dalia"; Piper angustifolium "matico".

Esta especie fue citada por Vilca \& Reyes (1999) para el Callejón de Huaylas. En el Perú ha sido registrado por Wille (1943), Alata (1973), Valencia \& Cárdenas (1973), Valencia et al. (1975), Ortiz (1980), Da Silva et al. (1980), Grados \& Ortiz (2004) y Delfino (2005) en diversos hospederos.

Nasonovia ribisnigri (Mosley 1841)

Distribución y Hospederos: Yungay. Lactuca sativa "lechuga".

En el Perú fue citada por Valencia et al. (1975) y Delfino (2005).

Rhopalosiphoninus latysiphon (Davidson 1912)

Distribución y Hospederos: Huaraz. Solanum tuberosum "papa".

En el Perú ha sido citada por Ortiz (1973), Valencia et al. (1975) y Grados \& Ortiz (2004), en diversos hospederos.

Sitobion avenae (Fabricius 1775)

Distribución y Hospederos: Huaraz; Carhuaz; Yungay y Huaylas. Triticum aestivum "trigo"; Hordeum vulgare "cebada"; Zea mays "maíz".

Fue citada para el Callejón de Huaylas por Vilca \& Reyes (1999). En el Perú ha sido mencionada por Valencia et al. (1975), Grados \& Ortiz (2004) y Delfino (2005), sobre especies de Poaceae.

Uroleucon ambrosiae (Thomas 1878)

Distribución y Hospederos: Huaraz; Carhuaz; Yungay y Huaylas. Rosa sp. "rosal"; Helianthus annuus "girasol"; Bidens pilosa "amor seco"; Chrysanthemum sp. "crisantemo"; Taraxacum sp. "diente de león"; Taraxacum officinale "diente de león"; Bellis perennis "margarita"; Bouganvillea spectabilis "buganvilia"; Aster $\mathrm{sp}$.

Citada para el Perú por Da Silva et al. (1980) y Delfino (2005) en diversos hospederos.

Uroleucon sonchi (Linnaeus 1767)

Distribución y Hospederos: Huaraz. Lactuca sativa "lechuga".

En el Perú ha sido citada por Valencia \& Cárdenas (1973), Da Silva et al. (1980), Grados \& Ortiz (2004) y Delfino (2005) en especies de Asteraceae.

Wahlgreniella nervata (Gillette 1908) 
Distribución y Hospederos: Huaraz y Yungay. Rosa sp. "rosal".

Escajadillo \& Ortiz (1993) y Delfino (2005) la citaron para el Perú en rosal.

\section{SUBFAMILIA PENPHIGINAE}

Eriosoma lanigerum (Hausmann 1802)

Distribución y Hospederos: Huaraz y Carhuaz. Malus domestica "manzano"; Pyrus communis "peral"; Cydonia oblonga "membrillero".

Fue citada para el Callejón de Huaylas por Vilca \& Reyes (1999), en manzano. En el Perú ha sido mencionada por Wille (1943), Alata (1973), Valencia et al. (1975), Grados \& Ortiz (2004) y Delfino (2005) en el mismo hospedero.

\section{SUBFAMILIA DREPHANOSIPHONINAE}

Therioaphis trifolii (Monell 1882)

Distribución y Hospederos: Huaraz; Carhuaz y Yungay. Medicago sativa "alfalfa".

Fue citada para el Callejón de Huaylas por Vilca \& Reyes (1999) en alfalfa como Therioaphis maculata. En el Perú ha sido citada por Ortiz \& Rubin de Celis (1992), Grados \& Ortiz (2004) y Delfino (2005) en alfalfa.

\section{SUBFAMILIA LACHNINAE}

Tuberolachnus salignus (Gmelin 1790)

Distribución y Hospederos: Yungay. Salix sp. "sauce".

Citada para el Perú por Alata (1973), Valencia et al. (1975) y Delfino (2005) en diversos hospederos.

\section{Conclusiones.}

- Las cuatro provincias del Callejón de Huaylas presentan una alta diversidad de áfidos: 26 especies en Huaraz, 23 en Carhuaz, 23 en Yungay y 18 en Huaylas.

- Se determinan 31 especies de áfidos ubicadas en 4 subfamilias y 5 tribus, en 52 especies de plantas, 19 familias botánicas, 45 géneros para el Callejón de Huaylas. Se establecen 123 asociaciones áfidoplanta.

- Se registran por primera vez para el Callejón de Huaylas las siguientes 18 especies de áfidos: Acyrthosiphon kondoi Shinji 1938, Aphis cytisorum Hartig 1841, Aphis fabae Scopoli 1763, Aphis helianthi Monell 1879, Aphis nerii Boyer de Fonscolombe 1841, Aphis spiraecola Patch 1914, Brachycaudus schwartzi (Börner 1931), Capitophorus elaeagni (Del Guercio 1894), Hyalopterus pruni (Geoffroy 1762), Metopolophium dirhodum (Walker 1849), Myzus ornatus Laing 1932, Nasonovia ribisnigri (Mosley 1841), Rhopalosiphoninus latysiphon (Davidson 1912), Rhophalosiphum padi (Linnaeus 1758), Tuberolachnus salignus (Gmelin 1790), Uroleucon ambrosiae (Thomas 1878), Uroleucon sonchi (Linnaeus 1767) y Wahlgreniella nervata (Gillette 1908).
- Se cita por primera vez en el Perú a las especies Aphis helianthi y Aphis cytisorum.

- Se confirma a Aphis cytisorum como el áfido de la retama, y la especificidad de $A$. helianthi para especies de la familia Asteraceae.

- Brevicoryne brassicae tiene un amplio rango de hospederos, sobre 9 especies de plantas, 4 familias, 7 géneros.

La mayor diversidad de especies de áfidos se encuentra en plantas de las familias Asteraceae, Fabaceae, Solanaceae y Rosaceae.

\section{Literatura citada.}

Alata J. 1973. Lista de insectos y otros animales dañinos a la agricultura en el Perú. Ministerio de Agricultura, Dirección General de Investigación Agraria. Lima.

Blackman R.L. \& Eastop V.F. 1984. Aphids on the world`s Crops: An Identification Guide. John Wiley \& Sons Ltd. Chichester, England.

1994. Aphids on the world's Trees: An Identification Guide. CAB Internacional and The Natural History Museum. London, U.K.

2006. Aphids on the World's Herbaceous Plants and Shrubs. Vol I-II. John Wiley \& Sons Ltd. Chichester.

2007. Taxonomic issues. Pags: 1-29. In: Van Emdem, H. F. and Harrington, R., Eds. Aphids as Crop Pests. CAB International. Wallingford.

Carver M. 1999. Uroleucon sonchi (Linnaeus) (Hemiptera: Aphididae) in Australia. Australian Journal of Entomology. 38: 314-317.

Cermeli M. 1984. Clave para la identificación de áfidos capturados en trampas en Venezuela. FONAIAP Centro Nacional de investigaciones Agronómicas. Serie A No 2-02, Maracay. Venzuela.

Da Silva T., Ortiz M. \& Ojeda D. 1980. Aphididae (Homoptera) del departamento de Lambayeque. Rev. Per. Ent. 23(1): 121-123.

Delfino M.A. 2005. Inventario de las relaciones áfido-planta en el Perú. Ecología Aplicada (Lima). 4 (1-2): 143-148.

Díaz W. \& Lozada P.W. 2007. Primer reporte de Brachycaudus schwartsi (Börner) (Hemiptera: Aphididae). Rev. Per. Biol. 14(2): 303.

Eastop V.F. \& Van Emden H.F. 1972. The insect material. Pp. 1-45. In: Emden, H.F. ed. Aphid technology. Academic Press. London.

Escajadillo C. \& Ortiz M. 1993. El áfido Wahlghreniella nervata, nuevo registro para el Perú y tres áfidos adicionales para Ica. Resumenes XXXV Convención Nacional de Entomología. Arequipa, Perú. : 42

Fribourg C.E. 2007. Virus, viroides y mollicutes de las plantas cultivadas en el Perú. Servicio y Publicidad S.A.C. Lima.

Grados J. \& Ortiz M.S. 2004. Los áfidos (Homoptera: Aphididae) y sus hospederos en el monte ribereño del Río Rímac. Rev. Per. Ent. 44: 1-10.

Heie O.E. 1980. The Aphidoidea (Hemiptera) of Fennoscandia and Denmark. I. General part. The families Mindaridae, Hormaphididae, Thelaxidae, Anoeciidae, and Pemphigidae. Fauna Entomologica Scandinavica. 9: 1-236. 
1982. The Aphidoidea (Hemiptera) of Fennoscandia and Denmark. II. The family Drephanosiphidae. Fauna Entomologica Scandinavica. 11: 1-176.

1986. The Aphidoidea (Hemiptera of Fennoscandia and Denmmark. III. Family Aphididae: subfamily Pterocommatine \& tribe Aphidini of subfamily Aphidinae. Fauna Entomologica Scandinavica. 17: 314

Heikinheimo O. 1988. Mounting techniques, aphid collections. Pp. 31-44. In: Minks, A. K. and Harrewijn, eds. Aphids. Their biology, natural enemies and control. Vol B. Elsevier. Amsterdam.

Holman J. 1974. Los áfidos de Cuba. Instituto Cubano del libro. La Habana.

2009. Host Plant Catalog of Aphids. Palaearctic Region. Springer. $1216 \mathrm{p}$.

Katis N.L., Tsitsipis J.A., Stevens, M. \& Powell G. 2007. Transmission of plant viruses. Pags. 353-390. In: Van Emdem, H. F. and Harrington, R., Eds. 2007. Aphids as Crop Pests. CAB International. Wallingford.

Klingauf F.A. 1987. Host plat finding and acceptance. Pp. 209-223. In: Minks, A. K. and Harrewijn, eds. Aphids. Their biology, natural enemies and control. Vol A. Elsevier. Amsterdam.

Ministerio de Agricultura. 2007. Encuesta por muestreo (EPM). Resultados region Sierra 2006. Departamento: Ancash. Dirección General de Información Agraria.

Nuñez E. 1993. Insectos del algarrobo (Prosopis sp.) en el Perú: Costa Norte (Piura) y Costa central (Ica): Rev. Per. Ent. 36: 69-83.

Ortiz M. 1973. Rophaloshiponinus latysiphon (Davidson), un áfido de sifúnculos peculiares. Rev. Per. Ent. 16: 125-126.

1980. Aphididae (Homoptera) procedentes de ceja de selva: Tingo María (Huánuco-Perú). Rev. Per. Ent. 23(1): 119-120.

Ortiz M., Rodríguez A.C. \& Sarmiento J. 1981. Los áfidos (Homoptera: Aphididae) de la cebada (Hordeum vulgare L.) en la sierra y costa centrales del Perú. Rev. Per. Ent. 24 (1): 133-136.
Ortiz M. \& Raven K.G. 1990. Ocho nuevos registros de Aphididae (Homoptera) para el Perú. Rev. Per. Ent. 33: 123-124.

Ortiz M. \& Rubín de Celis V. 1992. Therioaphis trifolii (Monell) (Homoptera: Aphididae), nuevo registro para el Perú. Rev. Per. Ent. 35: 51-52.

Pike S.K., Boydston L.L. \& Allison D.W. 2003. Aphids of Western North America North of Mexico. Washington State University.

Quisenbery S.S. \& Xinzhi N. 2007. Feeding injury. Pp. 331352. In: Van Emdem, H. F. and Harrington, R., Eds. 2007. Aphids as Crop Pests. CAB International. Wallingford.

Remaudière G. 1992. Une méthode simplifiée de montage des aphides et autres petits insectes dans le baumes du Canada. Rev. Fr. Ent. 14(4): 185-186.

Remaudière G. \& Remaudière M. 1997. Catalogue des Aphididae du Monde / Catalogue of the World's Aphididae. Homoptera Aphidoidea. INRA Editions. Versailles.

Sánchez V.G. \& Vergara C. 2002. Manual de Prácticas de Entomología Agrícola. Departamento de Entomología. Universidad Nacional Agraria La Molina. Lima- Perú.

Valencia L. \& Cárdenas N. 1973. Los áfidos (Homóptera: Aphididae) del valle de Ica, sus plantas hospederas y enemigos naturales. Rev. Per. Ent. 16(1): 6-14.

Valencia L.V., Guerra C. \& Gutarra F. 1975. Los áfidos (Homóptera: Aphididae) del valle del Mantaro, sus plantas hospederas y enemigos naturales. Rev. Per. Ent. 18(1): 90-97.

Vilca K. \& Reyes E. 1999. Identificación de áfidos (Homoptera: aphididae) y sus parasitoides en el Callejón de Huaylas, Ancash, Perú. Rev. Per. Ent. 41: 57-60.

Voegtlin D., Villalobos W., Sánchez M.V., Saborío G. \& Rivera C. 2003. Guía de los Afidos Alados de Costa Rica. Revista de Biología Tropical. Vol. 51 (2).

Wille J. 1943. Entomología Agrícola del Perú. Junta de sanidad vegetal. Dirección de Agricultura, Ministerio de Agrícultura. Lima, Perú.

\footnotetext{
${ }^{1}$ Universidad Nacional "Santiago Antunez de Mayolo", Apartado Postal 75, Huaraz-Perú, karinavilcamallqui@hotmail.com

${ }^{2}$ Universidad Nacional Agraria La Molina, Apartado Postal 456 Lima-Perú, cvc@lamolina.edu.pe
} 